\title{
WEIERSTRASS WEIGHT AND DEGENERATIONS
}

\author{
R. F. LAX
}

\begin{abstract}
It is shown that as a family of projective smooth curves degenerates to an irreducible Gorenstein curve the Weierstrass weight at a point $P$ on the limit curve is the sum of the Weierstrass weights at points on the smooth curves converging to $P$.
\end{abstract}

C. Widland [8] extended the classical notion of Weierstrass point to integral, projective Gorenstein curves. We considered Weierstrass points of invertible sheaves on such curves in [5] and showed that a singular point is always a Weierstrass point of high weight of any invertible sheaf with at least two linearly independent global sections. We remarked that this may be interpreted to mean that as a family of smooth curves degenerates to an irreducible Gorenstein curve, then many of the Weierstrass points tend towards the singularities. Our goal in this note is to make this statement precise.

We work over C. By a "point" of a scheme, we mean a closed point. If $X$ is a scheme of finite type over $\mathbf{C}$, then we denote the associated complex space by $X_{a n}$. We wish to thank Augusto Nobile and Peter Stiller for helpful conversations.

Let $S$ be an integral, noetherian scheme over $\mathbf{C}$ and let $\pi: \chi \rightarrow S$ be a family of Gorenstein curves of arithmetic genus $g \geq 2$. By this we will mean that $\pi$ is a flat, proper morphism whose geometric fibers are integral Gorenstein curves of arithmetic genus $g$. By the theory of duality of coherent sheaves [4, V.9.7 and VII.4 (p. 388)], there is a canonical invertible sheaf $\omega=\omega_{\chi / S}$ on $\chi$ whose restriction to a fiber $\chi_{s}$ is the sheaf of dualizing differentials on that curve.

Let $\mathcal{L}$ be an invertible sheaf on $\chi$. Then $\mathcal{L}$ is flat over $S$. Assume that $\operatorname{dim} h^{0}\left(\mathcal{X}_{s}, \mathcal{L}_{s}\right)=r>0$ for all $s \in S$. Then $\pi_{*} \mathcal{L}$ is locally free of rank $r$. We define below an effective Cartier divisor $\mathcal{W}(\mathcal{L})$ on $\chi$. We will also use $\mathcal{W}(\mathcal{L})$ to denote the associated locally principal closed subscheme of $\mathcal{X}$. We note that the subscheme $\mathcal{W}(\mathcal{L})$ may not be reduced. The points of $\mathcal{W}(\mathcal{L})$ are the $\mathcal{L}$-Weierstrass points of $\chi / S$. The $\omega^{\otimes n}$-Weierstrass points are called Weierstrass points of order $n$ and the $\omega$-Weierstrass points are simply called Weierstrass points.

Suppose $(s, P) \in X$ and let $U$ be an open neighborhood of $(s, P)$ such that

(1) There are sections $\phi_{1}, \ldots, \phi_{r}$ of $\mathcal{L}$ whose restrictions to $\chi_{s^{\prime}}$ form a basis for $H^{0}\left(\mathcal{X}_{s^{\prime}}, \mathcal{L}_{s^{\prime}}\right)$ for all $s^{\prime} \in \pi(U)$.

(2) $\mathcal{L}(U)$ is a free $O_{x}(U)$-module generated by $\psi$.

(3) $\omega_{\chi / S}(U)$ is a free $O_{\chi}(U)$-module generated by $\tau$.

Define $F_{i, j} \in \Gamma\left(U, O_{\chi}\right)$ by

$$
\begin{aligned}
\phi_{j} & =F_{1, j} \psi \quad \text { for } j=1, \ldots, r, \\
d F_{i-1, j} & =F_{i, j} \tau \quad \text { for } i=2, \ldots, r \text { and } j=1, \ldots, r,
\end{aligned}
$$

Received by the editors May 15, 1986 and, in revised form, June 27, 1986.

1980 Mathematics Subject Classification (1985 Revision). Primary 14H15.

Key words and phrases. Weierstrass point, Gorenstein curve. 
where we view $d F_{i-1, j}$ as a rational relative 1 -form regular on $U$. Then $\mathcal{W}(\mathcal{L})$ is given in $U$ as the scheme of zeros of

$$
f_{U}=\operatorname{det}\left[F_{i, j}\right], \quad i, j=1, \ldots, r .
$$

It is easy to see that this definition is independent of the choices we have made above. The fiber of $\mathcal{W}(\mathcal{L})$ over $s \in S$ consists of the $\mathcal{L}_{s}$-Weierstrass points on $\chi_{s}$ as defined in [5].

If $(s, P) \in \mathcal{X}$, then $W_{\mathcal{L}_{s}}(P)$, the $\mathcal{L}_{s}$-Weierstrass weight of $P$ (see [5]), is the order of vanishing at $P$ of the restriction of $f_{U}$ to $\chi_{s}$, which equals

$$
\operatorname{dim} 0 /\left(f_{U} \mid x_{s}\right)
$$

where $O$ denotes the local ring of $P$ on $x_{s}$. Let $\tilde{O}$ denote the normalization of 0 and put $\delta=\operatorname{dim} \tilde{O} / 0$. The following result was shown in [5].

PROPOSITION 1. $W_{\mathcal{L}_{s}}(P) \geq \delta \cdot r \cdot(r-1)$. In particular, if $P$ is a singular point and $r>1$, then $P$ is an $\mathcal{L}_{s}$-Weierstrass point.

PROPOSITION 2. $\mathcal{W}(\mathcal{L})$ is a relative effective Cartier divisor.

ProOF. We must show that $\mathcal{W}(\mathcal{L})$ is flat over $S$. Since $\pi: \mathcal{W}(\mathcal{L}) \rightarrow S$ is proper and has finite fibers, it is finite. Hence, it suffices to show that every fiber of $\mathcal{W}(\mathcal{L})$ has the same length. But by [5, Proposition 1], the length of the fiber of $\mathcal{W}(\mathcal{L})$ over $s$ is $r \cdot \operatorname{deg}\left(\mathcal{L}_{s}\right)+(r-1) r(g-1)$ and the degree of $\mathcal{L}_{s}$ is constant over $S$ since $\chi(\mathcal{L})$ is constant over $S$ by $[6$, p. 50$]$.

We now consider the complex spaces associated to the above schemes and the induced map $\pi_{a n}: \mathcal{W}(\mathcal{L})_{a n} \rightarrow S_{a n}$. Then $\pi_{a n}$ is quasi-finite, hence finite. Also, note that since

$$
\operatorname{dim} 0 /\left(f_{U} \mid x_{s}\right)=\operatorname{dim} \hat{O} /\left(f_{U} \mid x_{s}\right)
$$

the multiplicity of a point on a fiber of $\pi$ is the same as the multiplicity of the point on the corresponding fiber of $\pi_{a n}$. Hence

PROPOSITION 3. $\pi_{a n}: \mathcal{W}(\mathcal{L})_{a n} \rightarrow S_{a n}$ is flat.

Suppose $(s, P) \in X$ and let $U^{\prime}$ be any neighborhood of $(s, P)$ in the complex topology. Then there exists a complex neighborhood $V \subseteq U^{\prime}$ of $(s, P)$ such that $V \cap X_{s}$ contains no $\mathcal{L}$-Weierstrass points of $\chi_{s}$ except possibly for $P$ and the map $\pi_{a n} \mid \mathcal{w ( L ) \cap V}$ is flat. So for any such neighborhood $V$ we have

THEOREM 1. $\sum_{Q \in \mathcal{X}_{s^{\prime}} \cap V} W_{\mathcal{L}_{s^{\prime}}}(Q)=W_{\mathcal{L}_{s}}(P)$ for all $s^{\prime} \in \pi(V)$.

COROLLARY 1 (upper semicontinuity of weight). For any $(s, P) \in \mathcal{X}$ there exists a neighborhood $V$ of $(s, P)$ in the complex topology such that

$$
W_{\mathcal{L}_{s^{\prime}}}(Q) \leq W_{\mathcal{L}_{s}}(P)
$$

for all $\left(s^{\prime}, Q\right) \in V$.

The above corollary is similar to the "principle of nondegeneracy" enunciated by Rauch [7] and is related to results of Arbarello [1]. If $\pi$ is smooth, then the corollary follows from results of Eisenbud and Harris [3]. 
THEOREM 2. Let $Z$ be an integral projective curve of arithmetic genus $g$ which is locally embeddable in the plane (e.g. having singularities consisting of nodes and/or cusps). Suppose $P \in Z$ is a Weierstrass point of order $n$. Then there exists a flat, proper map $\pi: X \rightarrow Y$ from a nonsingular surface $X$ to a nonsingular curve $Y$ and a point $y_{0} \in Y$ such that $Z=\chi_{y_{0}}$ and such that $P$ is the limit of Weierstrass points of order $n$ on nonsingular curves lying over a neighborhood of $y_{0}$. If $P$ is a singular point of $Z$, then there are at least two sequences of Weierstrass points on nonsingular curves lying over a neighborhood of $y_{0}$ which converge to $P$.

PROOF. The existence of such a family of curves was proven by G. Winters [9]. By replacing $Y$, if necessary, by an open subscheme containing $y_{0}$ we may assume that $Y$ is irreducible and all fibers of $\pi$ except $Z$ are nonsingular curves. The fact that a Weierstrass point $P$ of order $n$ on $Z$ is the limit of Weierstrass points of order $n$ on nearby smooth curves follows from Theorem 1 . If $P$ is singular, then the $\left(\omega_{Z^{-}}\right)$ Weierstrass weight of $P$ is at least $g(g-1)$, while the Weierstrass weight at a point on a smooth curve of genus $g$ is at most $g(g-1) / 2$. Hence, by Theorem 1 , given any complex neighborhood of $\left(y_{0}, P\right)$ in $\chi$, there exists a subneighborhood $V$ such that there must be at least two Weierstrass points on each fiber of $\left.\pi\right|_{V-(V \cap Z)}$.

We note that S. Diaz [2] has proven that "the generic nonseparating node on a uninodal stable curve [of arithmetic genus $g$ ] is a limit of exactly $(g-1) g$ Weierstrass points on nearby smooth curves."

\section{REFERENCES}

1. E. Arbarello, Weierstrass points and moduli of curves, Compositio Math. 29 (1974), 325-342.

2. S. Diaz, Exceptional Weierstrass points and the divisor on moduli space that they define, Mem. Ainer. Math. Soc. 56 (1985), no. 327.

3. D. Eisenbud and J. Harris, When ramification points meet, Invent. Math. 87 (1987), 485-493.

4. R. Hartshorne, Residues and duality, Lecture Notes in Math., vol. 20, Springer-Verlag, Berlin and New York, 1966.

5. R. F. Lax, On the distribution of Weierstrass points on singular curves, Israel J. Math. 57 (1987), 107-115.

6. D. Mumford, Abelian varieties, Oxford Univ. Press, Oxford, 1970.

7. H. E. Rauch, Weierstrass points, branch points and moduli of Riemann surfaces, Comm. Pure Appl. Math. 12 (1959), 543-560.

8. C. Widland, On Weierstrass points of Gorenstein curves, Ph.D. dissertation, Louisiana State University, 1984.

9. G. Winters, On the existence of certain families of curves, Amer. J. Math. 96 (1974), 215-228.

Department of Mathematics, Louisiana State University, Baton Rouge, LOUISIANA 70803 